\title{
A medication adherence- enhancing simulation intervention in pediatric cystic fibrosis
}

Journal of Child Health Care 202I, Vol. 0(0) I-15

(C) The Author(s) 2021

Article reuse guidelines: sagepub.com/journals-permissions DOI: 10.1177/13674935211013924 journals.sagepub.com/home/chc @SAGE

\author{
Guy Van Schandevyl' ${ }^{1}$, Georges Casimir ${ }^{2}$, and \\ Laurence Hanssens ${ }^{2}$
}

\begin{abstract}
Adherence to chronic pulmonary drugs in cystic fibrosis (CF) is suboptimal. We studied the feasibility and effectiveness of a multistep medication adherence-enhancing simulation intervention for pediatric CF, which was embedded in motivational interviewing and education. Product simulation experiments were performed by the children themselves, and they addressed adherence to mucolytics/hydrators and antibiotics. Dornase alfa-treated patients aged 7-13 years were included. We invited each patient and their parents to attend an interview. PowerPoint slides were presented and discussed. The final slide invited the patient to perform the simulation experiments, and, in so doing, they experienced what happens when they either do or do not take their medication. An educational film was applied as a summary tool. A patient-centered empathic counseling style was used. Two months later, the child and their parents each completed a different anonymous questionnaire. Overall, 21 patients were included. Parents rated the means of communication and improvement in their child's motivation as very satisfactory. Children highly appreciated the experiments they performed. They often answered two questions on dornase alfa correctly and associated knowledge with adherence. Our results suggest that experiential simulation-based learning is extremely appropriate, and that this multistep intervention is feasible and effective in pediatric CF.
\end{abstract}

\footnotetext{
' Pediatric Cystic Fibrosis Center, Hôpital Universitaire des Enfants Reine Fabiola, Université Libre de Bruxelles (ULB), Brussels, Belgium

${ }^{2}$ Department of Pulmonology, Allergology and Cystic Fibrosis, Hôpital Universitaire des Enfants Reine Fabiola, Université Libre de Bruxelles (ULB), Brussels, Belgium

Corresponding author:

Guy Van Schandevyl, Pediatric Cystic Fibrosis Center, Hôpital Universitaire des Enfants Reine Fabiola, Université Libre de Bruxelles (ULB), Avenue Crocq 15, Bruxelles 1020, Belgium, Europe.

Email: guy.vanschandevyl@huderf.be
} 


\section{Keywords \\ Cystic fibrosis, child, adherence, product simulation, education, experiential learning, motivational interviewing, clinical pharmacy}

\section{Introduction}

Cystic fibrosis (CF) is a systemic autosomal recessive disease caused by mutations in the gene that encodes the CF transmembrane conductance regulator (CFTR) protein, which functions as an anion channel (Elborn, 2016). CF transmembrane conductance regulator dysfunction in the airways results in mucous abnormalities, reduced airway surface liquid (ASL) hydration, suboptimal mucociliary clearance, mucus retention, infection, inflammation, and lung injury (Elborn, 2016). Airway clearance drugs that improve mucociliary clearance (e.g., dornase alfa [Pulmozyme ${ }^{\circledR}$, Roche Pharma AG, D-79639 Grenzach-Wyhlen, Germany] and hypertonic saline), inhaled antibiotics (e.g., colistin, tobramycin, and aztreonam), and oral azithromycin, all of which are often combined, aim to improve lung function and reduce the frequency of pulmonary exacerbations (Elborn, 2016).

In 2017, the Belgian national registry included 1319 people with $\mathrm{CF}, 37 \%$ of whom were under 18 years old (Zolin et al., 2019).

The World Health Organization (2003) defines adherence to long-term therapy as "the extent to which a person's behavior - taking medication, following a diet, or executing lifestyle changescorresponds with agreed recommendations from a health care provider," and it states that adherence to long-term therapy for chronic illnesses in developed countries averages about $50 \%$.

Low adherence to CF medication therapy is associated with an increased risk of respiratory exacerbation, hospitalization, and longer length of stays (Eakin and Riekert, 2013).

Adherence to chronic pulmonary drugs in CF patients is suboptimal, and poor adherence is associated with worse lung-health outcomes (Eakin and Riekert, 2013; Quittner et al., 2014; Riekert et al., 2015). Thus, effective interventions are needed to improve medication adherence in CF (Eakin and Riekert, 2013; Quittner et al., 2014; Riekert et al., 2015). Poor adherence to inhaled therapies particularly needs to be addressed (Duff and Latchford, 2010a; Castellani et al., 2018).

Variables associated with adherence include individual and family factors, medication regimens, measurement issues, knowledge, and interaction with healthcare professionals, whereas organizational, psychoeducational, psychotherapeutic, and motivational methods that have been shown to improve adherence (Duff and Latchford, 2010b). Nicolais et al. (2019) used interviews with a sample of parents and adolescents, thereby identifying overall nine facilitators of treatment adherence in CF.

Miller and Rollnick (2013), the cofounders of motivational interviewing (MI), define MI as a collaborative, goal-centered style of communication. Motivational interviewing is designed to create an atmosphere of acceptance and compassion that strengthens personal motivation and commitment toward a defined goal by eliciting and exploring the individual's own reasons for change.

A meta-analysis by Hettema et al. (2005) revealed strong evidence for MI in addictive and health behaviors. Another meta-analysis found that MI demonstrated a significant and clinically relevant effect for various behavioral problems in about $75 \%$ of studies (Rubak et al., 2005). Rubak et al. (2005) thus concluded that MI outperformed traditional advice for a wide range of behavioral problems and diseases. However, a Cochrane review of interventions targeted to enhance 
medication adherence did not identify any common successful intervention characteristics among these studies (Nieuwlaat et al., 2014). A recent systematic review (Gesinde and Harry, 2018) concerning the use of $\mathrm{MI}$ in an effort to improve medication adherence for individuals with asthma pertained to 11 studies published between 2001 and 2016. These studies demonstrated that MI as a stand-alone method was not efficient, but they also indicated that MI coupled with other interventions (e.g., education, family support, technology use, or social media support) was shown effective in improving medication adherence. Therefore, Gesinde and Harry (2018) have recommended a multifaceted approach to improving asthma medication adherence. A Cochrane review of self-management education for CF found that the available evidence was of insufficient concerning quantity and quality to draw any firm conclusions (Savage et al., 2014).

Zobell et al. $(2017,2018)$ demonstrated that pharmacy services improved dornase alfa adherence, which was measured by means of the medication-possession ratio. Subsequently, this reduced the odds of hospitalization for pediatric CF patients. In this study, a standardized template was developed (e.g., medication reconciliation and education), medication adherence issues were followed by medication counseling, and potential barriers to adherence were discussed with the patient (Zobell et al., 2017). Sustainability of the positive impact of these pharmacy services was also demonstrated (Zobell et al., 2018). A specialist CF clinical pharmacist, working as part of the CF multidisciplinary team, was shown to play a significant role in supporting adherence (Thompson et al., 2016).

Methods to improve medication adherence in pediatric CF likely involve MI, education, or both. To our knowledge, coupling MI with specific experiential learning through product simulation experiments, which are performed by children with $\mathrm{CF}$, has never been thoroughly analyzed. The current study primarily attempts to fill this gap.

\section{Aim}

The aim of this study was to investigate the feasibility and effectiveness of a multistep medication adherence-enhancing simulation intervention, which was embedded in both MI and pediatric CF education.

\section{Materials and methods}

Product simulation experiments (https://youtu.be/rCFRSwN2biY) were developed by the clinical pharmacist in order to address adherence to mucolytics/hydrators and antibiotics.

All members of the CF care team had MI training in the past.

\section{Pre-study involving patients who only watch the educational film}

We performed a pre-study in which five CF patients were invited to watch the educational film (https://youtu.be/rCFRSwN2biY), individually and on a tablet, during their waiting time. This meant there was no motivational interview, and the patients did not perform the experiments themselves. During their next regular visit, these children exhibited very poor knowledge of what the film was all about and about what it sought to explain (through oral questions).

Due to this poor outcome, a multistep medication adherence-enhancing simulation intervention was implemented. The children with CF now did perform the experiments themselves. We hypothesized that this specific experiential learning type, namely, simulation-based learning, would be 
well accepted and well appreciated and thus be more effective than passive audiovisual learning. As a result, such an intervention would encourage many reflections on the experience, rendering it at the end extremely suitable for use in young CF patients.

\section{Inclusion and exclusion criteria}

All CF patients aged from 7 to 13 years and who were treated with dornase alfa were invited to participate in this study, which was conducted at the pediatric CF center of Queen Fabiola Children's University Hospital (QFCUH) from December 2017 until July 2018. Children with CF who were yet unable to read were excluded. Likewise, CF patients over 13 years of age were excluded because they could be included in a transitional project group.

Study participants were recruited by the CF clinical pharmacist during their regular visits at the pediatric CF center. The CF pharmacist has access to the medical files and to the weekly list of children with CF who would visit the center. As part of the CF care team, the pharmacist also participates in the weekly CF multidisciplinary team meeting.

\section{Multistep intervention}

To review several of each patient's medications, the CF pharmacist invited the patient and their (grand) parent(s) to attend an interview. The subject and purpose of the harmless product simulation experiments were explained, and the children were informed that they had been invited to perform the experiments themselves. While the interview was conducted in the presence of the (grand) parent(s), it was actually focused on the child. Emphasis was put on that the experiment was meant to be a constructive talk, rather than a test that needed to be done.

Motivational interviewing was usually scheduled during regular patient visits to the pediatric CF center and could be postponed at the patient's or parent's request. It preferably took place in the clinical pharmacist's office as the latter was outside the examining room.

A patient-centered empathic counseling style was applied, which involved actively listening to the patient. Product simulation experiments were preceded and also followed by MI and education.

\section{$\mathrm{MI}$ and education}

Motivational interviewing and education were started and ended with everyone disinfecting their hands using a sanitizer pump. Three PowerPoint slides were discussed. The first slide showed a cartoon of a pharmacist indicating a nonjudgmental, friendly approach, and the patient was asked about their medication needs. A second slide exhibited an illustration of the respiratory system, along with a few notes on CF problems, and proposed solutions. This presentation could discuss salt transport and channel modulators, ASL and mucolytics/hydrators, mucus clearance and physiotherapy, infection and antibiotics, inflammation, as well as anti-inflammatory drugs. The children were encouraged to ask questions when there was something they were unable to completely understand. These questions were answered/followed by patient-centered educational explanations, affirmations, reflections, or summary statements. The patients were similarly encouraged to find answers and solutions by themselves. A third slide explained the working mechanisms of the mucociliary escalator, bacteria capture, and viscosity. This last slide was designed to prompt further engagement with the simulation experiments to be performed by the children with CF. This was then followed by an educational film. 
Open-ended questions were asked to explore both knowledge and adherence and to better understand the child's perspective and beliefs. These questions could, for example, be as follows: "How and when do you wash and disinfect your hands?" "Why is hand hygiene essential?" What do germs look like?" "How would you describe the air that we breathe?" "How do we breathe?" "What happens to the germs that we breathe in?" "What is CF and what does it mean for you?" "What would you like to know about your medications?" "What medications do you take?"

\section{Simulation experiments}

Experiments that had been developed by the clinical pharmacist were then displayed, which were shown in an educational film (https://youtu.be/rCFRSwN2biY). The children then experimented with the products simulating mucus, mucolytics, and antibiotics in order to visualize and understand their real-life functions. In so doing, they experienced what happened when they either did or not did take their medication (Figures 1 and 2).

The experiments have been grounded in educational and MI principles. Open-ended questions were asked to explore knowledge, potential barriers, and facilitators to treatment adherence, ambivalence, and resistance. These questions, for example, were "How do we get rid of germs?" "What is mucus?" "What is mucus good for?" "What is viscosity?" "Why practice nasal lavage?" "What mucolytics and antibiotics do you take?" "When, how, and why do you take them?" "Why do you not take them?" "What does an average school day look like?" "How about weekends and holidays?" "When, how, and why is physiotherapy performed?" "Why is it difficult to...?" "What would you like to change?"

Individually tailored interventions, which use shared decision-making to overcome adherence barriers, were then proposed and discussed. These interventions possibly involved either simplifying, prioritizing treatments, or both options, providing a clear color-coded medication schedule, tips and tricks to avoid tastes and/or throat irritation due to medication, adapting dosing times, and switching to inhaled powder to overcome the embarrassment of using time-consuming aerosols.

Persistent ambivalence may have been due to unresolved adherence barriers (e.g., treatment burden and embarrassment) and the patient's wish to simultaneously feel better. However, the patient has experienced the disadvantages of partial or nonadherence in the simulation experiments, and the patient's own arguments for change are elicited and reinforced during these experiments. The pros and cons of behavioral change were then discussed, with the patient's freedom of choice fully respected. Although the patient was actually supported, it was ultimately the patient's responsibility to resolve their ambivalence (Figure 3).

In resistance cases, empathy and reflective listening should be applied. Confrontation should be avoided at all costs, whereas changes should not be imposed, but only simple advice be given.

\section{Educational film}

The pre-study educational film (https://youtu.be/rCFRSwN2biY) was applied as a summary tool after the pediatric CF patients had performed the simulation experiments. These experiments clearly showed what would occur if

1. the mucolytic was forgotten;

2. the mucolytic was forgotten, with only the antibiotic administered;

3. the mucolytic was administered, but the antibiotic forgotten; and 


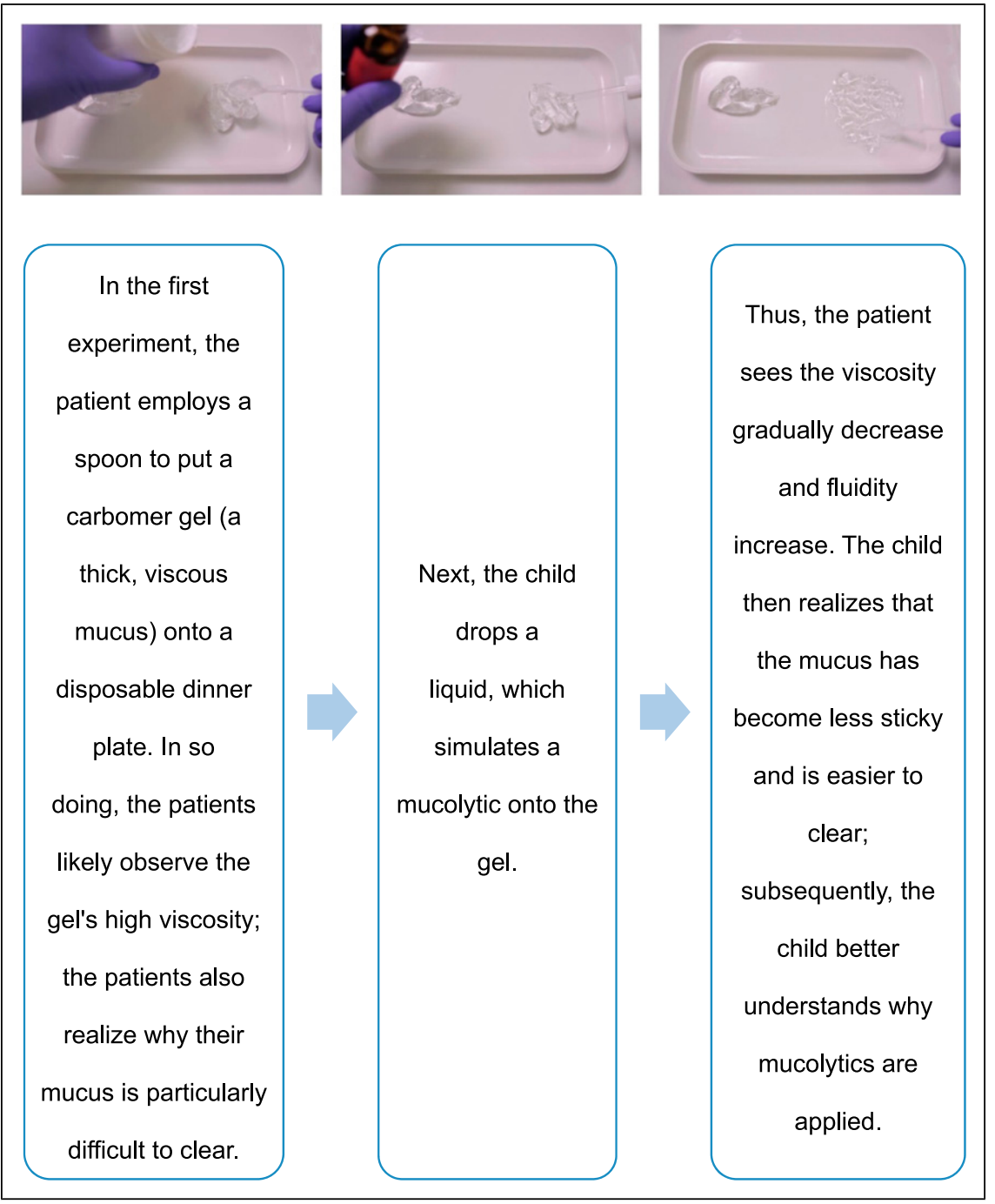

Figure I. First test setup.

4. both the mucolytic and antibiotic forgotten.

Patients were told that it was key to remember their medications, to use them correctly, and to administer the medications in the right order (first the mucolytic, followed by expectoration, and then the antibiotic).The child and their parents were not judged, though their body language and facial expressions often suggested what was actually happening in real life.

The pediatric $\mathrm{CF}$ patients were then empowered to make the connection between the simulation products and their real therapeutic experiences with, for example, dornase alfa, hypertonic saline, tobramycin, colistimethate, and aztreonam for inhalation. The relevance of physiotherapy, 




Figure 2. Second test setup.

expectoration, and nasal irrigation was also explored. Depending on the child's maturity, ongoing and future scientific developments like CFTR modulators and gene therapy could actually be discussed.

\section{Questionnaires}

At the MI's end, the child and their parents were informed that they would be invited to complete different short, anonymous questionnaires during their next visit to the CF center. It was emphasized that this would neither be a written test/examination nor involve any judgment, while the questionnaire was described as a follow-up that was primarily designed to improve CF teamwork. The children or their parents posted the questionnaire through a special letterbox at the CF secretariat.

The questionnaires were developed by the CF clinical pharmacist, discussed within and validated by the CF multidisciplinary team. The questions focused on communication, education, motivation, experiential learning through simulation experiments, knowledge, and adherence. Separate questionnaires were used for parents (Table 1) and children (Table 2). The parent questionnaire included seven items and the child questionnaire included three items, plus three true/false questions. Completed questionnaires were collected in the CF center about 2 months after the MI. 


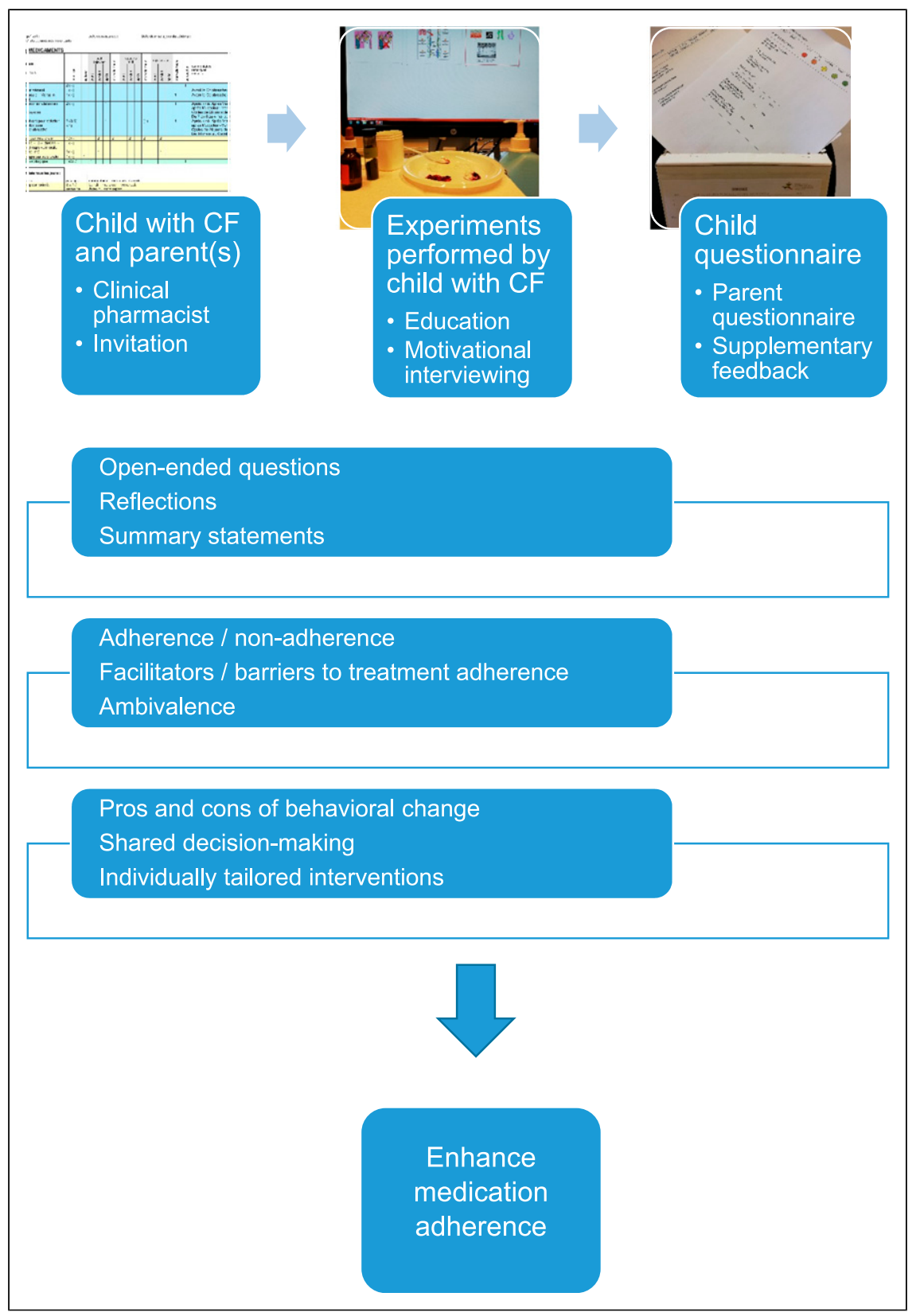

Figure 3. Multistep intervention flowchart. 
Table I. Parent questionnaire.

\begin{tabular}{|c|c|c|c|c|c|}
\hline & $\begin{array}{l}\text { Not at } \\
\text { all } \\
\text { satisfied }\end{array}$ & $\begin{array}{l}\text { Poorly } \\
\text { satisfied }\end{array}$ & $\begin{array}{l}\text { Average } \\
\text { Neutral }\end{array}$ & Satisfied & $\begin{array}{l}\text { Very } \\
\text { satisfied }\end{array}$ \\
\hline \multicolumn{6}{|l|}{$\begin{array}{l}\text { Means of communication: simulation experiments, } \\
\text { Powerpoint slides, and film }\end{array}$} \\
\hline \multicolumn{6}{|l|}{ Pharmacist's interactions with your child } \\
\hline \multicolumn{6}{|l|}{$\begin{array}{l}\text { Pharmacist's interactions with parents and/or } \\
\text { grandparents }\end{array}$} \\
\hline \multicolumn{6}{|l|}{ Explanations of cystic fibrosis and viscous mucus } \\
\hline \multicolumn{6}{|l|}{ Explanations of mucolytics and antibiotics } \\
\hline \multicolumn{6}{|l|}{$\begin{array}{l}\text { Explanations about the relevance of combining mucolytics } \\
\text { - expectoration (physiotherapy) - antibiotics }\end{array}$} \\
\hline $\begin{array}{l}\text { Improvement of your child's motivation to follow their } \\
\text { treatment (for example aerosol therapy) }\end{array}$ & & & & & \\
\hline
\end{tabular}

Table 2. Child questionnaire.

Your age: .. years
WHAT DO YOU THINK?
What do you think of the experiments you performed?
What do you think of the youtu.be video?
What do you think of the explanations by the pharmacist?
TRUE or FALSE?
Pulmozyme ${ }^{\circledR}$ makes the mucus become more fluid
If I am on antibiotics, I do not need to continue Pulmozyme ${ }^{\circledR}$ true false
aerosol therapy
If I understand better my treatment, I follow it better and forget true false
less often doing it

\section{Materials used for product simulation}

Mucus simulation: mucilago carbomeri, which is a clear, colorless, and odorless carbomer gel that is obtained via pharmaceutical compounding: R/carbomer $9801 \mathrm{~g}$, trometamol $1 \mathrm{~g}$, disodium EDTA $100 \mathrm{mg}$, propyleneglycol $10 \mathrm{~g}$, water (preserved with parabens) q.s. ad $100 \mathrm{~g}$.

Mucolytic simulation: a one-in-four dilution of glacial acetic acid in a dropper bottle.

Antibiotic simulation: colorants, such as cochineal red or riboflavin.

Infection simulation: a sachet of instant cappuccino or ground coffee, which is mixed into the gel. This setup is completed by using disposable dinner plates, teaspoons, and napkins. 


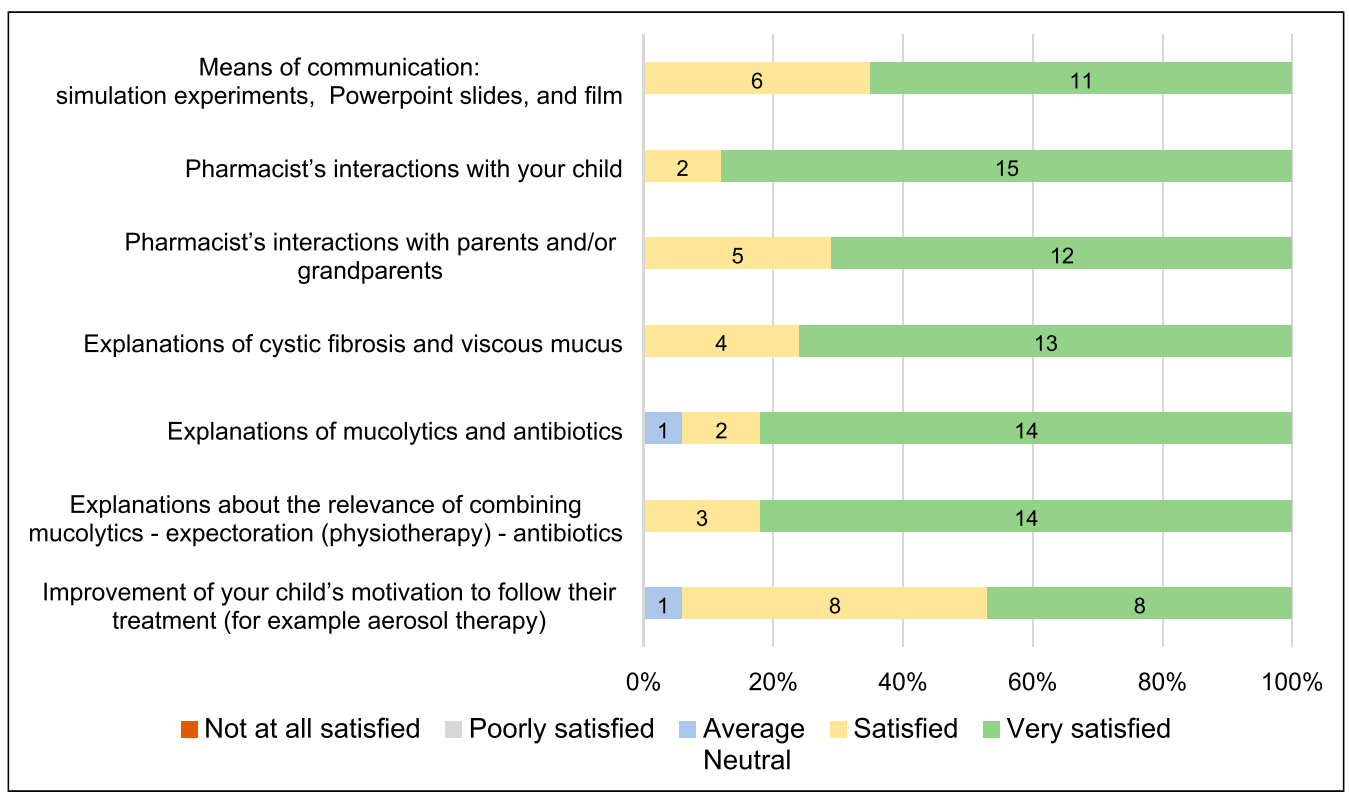

Figure 4. Parent questionnaire-results, $\mathrm{n}(\%)$.

\section{Results}

None of the children or their parents refused to participate in the MI or questionnaires. Results from the parent questionnaire (Figure 4) and child questionnaire (Figures 5 and 6) involved 17 parents and 21 children with CF (six boys and 15 girls), all of whom completed both the MI and questionnaires. Within this group of 21 children with $\mathrm{CF}$, there were four pairs of siblings with CF. The parents of the pairs of siblings with CF preferred to complete only one parent questionnaire. For one child, the MI was performed in the presence of their teacher, who also completed the parent questionnaire.

Results from the parent questionnaire (Figure 4) show that all seven investigated items were rated as average $(2 \%)$, satisfactory $(25 \%)$, or very satisfactory $(73 \%)$. No items were assessed as unsatisfactory or not at all satisfactory. The means of communication, interactions with the clinical pharmacist, the explanations, and improvement in the child's motivation to follow their treatment all obtained a positive review in the parent questionnaire.

Results from the child questionnaire (Figures 5 and 6) show that all three investigated items (the experiments, film, and explanations) were rated as follows: not good $(2 \%)$, average $(8 \%)$, great (30\%), or super! $(60 \%)$. None of the items were rated as bad $(0 \%)$. The experiments performed by the children themselves were rated particularly high.

Although the questionnaires were completed and collected at the CF center about 2 months after the MI, most of the children answered two questions about dornase alfa correctly. All of the children agreed with the statement "If I understand better my treatment, I follow it better and forget less often doing it." 


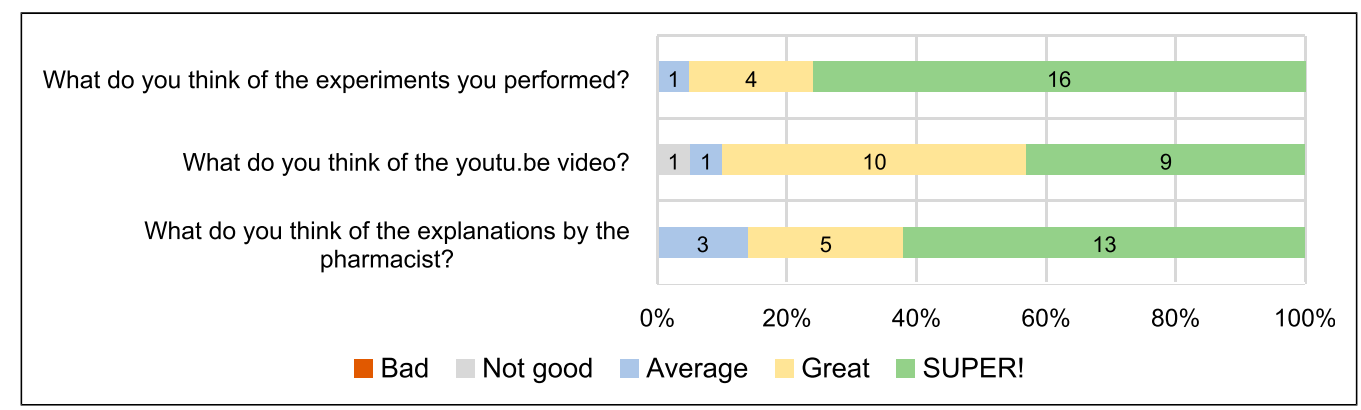

Figure 5. Child questionnaire-results, n (\%).

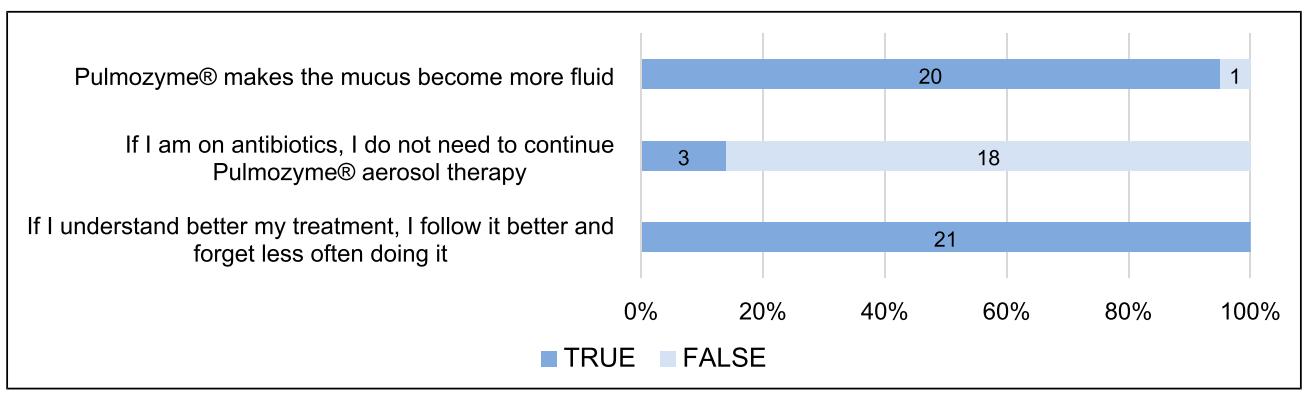

Figure 6. Child questionnaire-results, n (\%).

\section{Supplementary feedback}

Supplementary oral feedback was mostly obtained during the next regular visit. Sometimes it was obtained during hospitalization.

In addition to the questionnaires, the following unsolicited feedback was received from parents and/or children:

1. A 12-year-old child performed the simulation experiments in front of their class at school. The parents had previously asked for support with the material. The CF care team later received the teacher's evaluation and comments on the patient's presentation through email by the parents.

2. A 13-year-old child resumed ordering aztreonam for inhalation from the hospital pharmacy, having ignored basic knowledge of their treatment. However, their mother had detailed knowledge.

3. A 12-year-old child tried to repeat the simulation experiments at home.

4. The grandfather of an eight-year-old child asked for further written instructions for $\mathrm{CF}$ treatment.

5. The mother of an eight-year-old child said that it took her a long time to explain CF to her child, but that this visual and hands-on approach proved to be much faster and more effective than her own.

6. The link to the video was frequently requested.

7. Sometimes, the video was shown to and discussed with other family members at home. 
8. Several parents reevaluated the timing of their drug administration and/or physiotherapy, and treatment was sometimes adapted or simplified using shared decision-making.

9. Parents often reported that they also learned a lot.

\section{Discussion}

Modi et al. (2006) assessed rates of adherence in 37 children with CF aged between six and 13 years using four different measurement methods: self-reporting, diary data, pharmacy refill histories, and electronic monitors. The more objective measures displayed rates of overall adherence below 50\% for children with CF (Modi et al., 2006). In a study by Quittner et al. (2014), adherence to chronic respiratory medications was measured based on pharmacy refill histories for $3287 \mathrm{CF}$ patients, including both children ( $\geq 6$ years) and adults. Average drug-specific adherence ranged from $40 \%$ to 57\% (Quittner et al., 2014). Adherence among patients aged 6-10 years was significantly higher than among other age groups, which possibly indicates parental support (Quittner et al., 2014).

Nicolais et al. (2018) identified gaps in parents' knowledge, which may lead to poor adherence. The authors have recommended that parents annually complete the Knowledge of Disease Management-CF measure (KDM-CF-P), which focuses on the day-to-day management of CF. This assessment could facilitate identification and remediation of these gaps (Nicolais et al., 2018). Higher maternal education was associated with better scores on both treatment information and management scales (Nicolais et al., 2018).

Providing pictorial and audiovisual material could be considered to overcome limited literacy and its negative impact on medication adherence (Brown and Bussell, 2011).

Passive audiovisual learning, in which children only watch the educational film, was evaluated prior to the study and showed poor learning retention. In contrast, the product simulation experiments that were performed by the children with CF themselves seem to be more effective than only watching the educational film on a tablet. However, it is relevant to note that these experiments formed part of the larger multistep intervention. The specific type of experiential learning, namely, simulation-based learning, is a means of active learning. This type of learning was highly appreciated, well remembered, and induced many reflections, which all indicate that this learning is extremely appropriate for use in these young CF patients.

Results of our questionnaires all support our intervention strategy which combines MI and education to improve medication adherence.

No one-size-fits-all intervention is available to improve medication adherence (Nieuwlaat et al., 2014). However, a multifaceted approach that combines MI and other interventions has demonstrated effectiveness, and it is recommended to enhance medication adherence (Gesinde and Harry, 2018). Our complex intervention is also multifaceted and can be described as a CF medication adherence-enhancing simulation intervention that is embedded in MI and education. Motivational interviewing and education support the simulation experiments and vice versa.

Lin et al. (2017) found no significant associations between disease- and treatment-related knowledge and self-reported medication adherence in adults CF patients. Our study suggests an association between knowledge and adherence in children with $\mathrm{CF}$, given that they all agreed on the statement: "If I understand better my treatment, I follow it better and forget less often doing it."

Next steps could include broadening the age range, adding more items/questions to the questionnaires, and comparing drug ordering before and after the MI. Future research should also include longer-term follow-up. 
Electronic monitoring of, for example, pharmacy records or data collected by nebulizers could objectively measure medication adherence. However, this should be used with care and should not jeopardize the partnership between patients, parents, and the multidisciplinary team.

As parents' knowledge of CF can also influence their children's adherence, we recommend that they are present during the interview. We also recommend the introduction of the parent knowledge assessment (Nicolais et al., 2018) to identify and address gaps in parents' knowledge.

With the arrival of new promising personalized medicines, maintaining well-being by enhancing adherence is essential, especially for these young CF patients.

\section{Study limitations}

Several limitations must be considered when evaluating this study, such as its small sample size, adherence measurement issues, and the disadvantages of using questionnaires (e.g., dishonesty, subjectivity, misinterpretation, not taken seriously enough, social desirability bias, or researcher imposition). The gender ratio was also unbalanced as the study included 15 girls and only six boys. A follow-up of 2 months after the experiment could be considered relatively short. Further research should include a statistical data analysis based on more data, more study participants, and an increased study duration. Although our multistep intervention was extensively described, there may be a lack of clarity due to its complex nature.

\section{Implications for practice}

The present study couples MI with specific experiential learning, which may provide a useful framework for a multistep intervention that healthcare providers can use to enhance medication adherence in pediatric CF. Simulation-based learning is most suitable for use in young CF patients.

A synergistic combination of MI and education, with active involvement of the patient through experiential learning, is proposed to enhance medication adherence.

Motivational interviewing training should be provided for all members of the care team.

Within the context of a multidisciplinary team, a clinical pharmacist can play a significant role in supporting adherence.

\section{Conclusion}

The results suggest that our multistep medication adherence-enhancing simulation intervention in pediatric CF is feasible, effective, and well accepted by children with $\mathrm{CF}$ and their parents alike. Simulation experiments could provide promising new tools to improve knowledge and adherence.

Drugs do not help when not taken. Our intervention strategy could also be of interest in other chronic illness groups with poor medication adherence.

\section{Educational film}

"Cystic fibrosis: the use of antibiotics and mucolytics." https://youtu.be/rCFRSwN2biY (English). https://youtu.be/zQFuxKpwQdE (French). https://youtu.be/LH6BPpSw5Zc (Dutch). 
This film was made at the pediatric CF center at the Queen Fabiola Children's University Hospital (QFCUH), Brussels, Belgium.

\section{Declaration of Conflicting Interests}

The author(s) declared no potential conflicts of interest with respect to the research, authorship, and/or publication of this article.

\section{Ethical approval}

The Ethics Committee of the QFCUH, Brussels, Belgium, provided approval for this study (approval number $85 / 18$ ). Written informed consent was obtained from the patients' parents, and written informed assent was obtained from children $\geq 10$ years of age.

\section{Funding}

The author(s) received no financial support for the research, authorship, and/or publication of this article.

\section{ORCID iD}

Guy Van Schandevyl (1) https://orcid.org/0000-0001-7453-1646

\section{References}

Brown MT and Bussell JK (2011) Medication adherence: WHO cares? Mayo Clinic Proceedings 86: 304-314.

Castellani C, Duff AJA, Bell SC, et al. (2018) ECFS best practice guidelines: the 2018 revision. Journal of Cystic Fibrosis 17: 153-178.

Duff AJA and Latchford GJ (2010a) Inhaled medication and inhalation devices for lung disease in patients with cystic fibrosis: poor adherence and the need to address it. Journal of Cystic Fibrosis 9: 455-456.

Duff AJA and Latchford GJ (2010b) Motivational interviewing for adherence problems in cystic fibrosis. Pediatric Pulmonology 45: 211-220.

Eakin MN and Riekert KA (2013) The impact of medication adherence on lung health outcomes in cystic fibrosis. Current Opinion in Pulmonary Medicine 19: 687-691.

Elborn JS (2016) Cystic fibrosis. Lancet 388: 2519-2531.

Gesinde B and Harry S (2018) The use of motivational interviewing in improving medication adherence for individuals with asthma: a systematic review. Perspectives in Public Health 138: 329-335.

Hettema J, Steele J and Miller WR (2005) Motivational interviewing. Annual Review of Clinical Psychology 1: $91-111$.

Lin AH-A, Kendrick J, Wilcox PG, et al. (2017) Patient knowledge and pulmonary medication adherence in adult patients with cystic fibrosis. Patient Preference and Adherence 11: 691-698.

Miller WR and Rollnick S (eds) (2013). Motivational interviewing: helping people change. 3rd edition. New York, USA: Guilford Press.

Modi AC, Lim CS, Yu N, et al. (2006) A multi-method assessment of treatment adherence for children with cystic fibrosis. Journal of Cystic Fibrosis 5: 177-185.

Nicolais CJ, Bernstein R, Riekert KA, et al. (2018) Parent knowledge of disease management in cystic fibrosis: assessing behavioral treatment management. Pediatric Pulmonology 53: 162-173.

Nicolais CJ, Bernstein R, Saez-Flores E, et al. (2019) Identifying factors that facilitate treatment adherence in cystic fibrosis: qualitative analyses of interviews with parents and adolescents. Journal of Clinical Psychology in Medical Settings 26: 530-540.

Nieuwlaat R, Wilczynski N, Navarro T, et al. (2014) Interventions for enhancing medication adherence. The Cochrane Database of Systematic Reviews 11: CD000011. 
Quittner AL, Zhang J, Marynchenko M, et al. (2014) Pulmonary medication adherence and health-care use in cystic fibrosis. Chest 146: 142-151.

Riekert KA, Eakin MN, Bilderback A, et al. (2015) Opportunities for cystic fibrosis care teams to support treatment adherence. Journal of Cystic Fibrosis 14: 142-148.

Rubak S, Sandbæk A, Lauritzen T, et al. (2005) Motivational interviewing: a systematic review and metaanalysis. British Journal of General Practice 55: 305-312.

Savage E, Beirne PV, Ni Chroinin M, et al. (2014) Self-management education for cystic fibrosis. The Cochrane database of systematic reviews 9, CD007641.

Thompson K, Shaw N, Bentley S, et al. (2016) Role of the clinical pharmacist in the management of CF. Available at: https://hospitalpharmacyeurope.com/role-of-the-clinical-pharmacist-in-the-management-ofcf/ (accessed 9 September 2019).

World Health Organization (2003). Adherence to long-term therapies: evidence for action. Geneva, Switzerland: Publications of the World Health Organization. Available at: https://www.who.int/chp/ knowledge/publications/adherence_full_report.pdf?ua=1 2003 (accessed 9 September 2019).

Zobell JT, Schwab E, Collingridge DS, et al. (2017) Impact of pharmacy services on cystic fibrosis medication adherence. Pediatric Pulmonology 52: 1006-1012.

Zobell JT, Collingridge DS and Asfour F (2018) Impact of pharmacy services on cystic fibrosis medication adherence: update. Pediatric Pulmonology 53: 694-695.

Zolin A, Orenti A, van Rens J, et al. (2019) European Cystic Fibrosis Society Patient Registry Annual Report 2017. Available at: https://www.ecfs.eu/sites/default/files/general-content-images/working-groups/ecfspatient-registry/ECFSPR_Report2017_v1.3.pdf (accessed 28 September 2019). 